\title{
IncRNA_Mdeep: an alignment-free predictor for long non-coding RNAs identification by multimodal deep learning
}

\author{
CURRENT STATUS: UNDER REVISION \\ BMC Bioinformatics $\mathbf{B}$ BC Series \\ Xiao-Nan Fan \\ Northwestern Polytechnical University \\ ๑anxn0223@163.comCorresponding Author \\ Shao-Wu Zhang \\ Northwestern Polytechnical University \\ Song-Yao Zhang \\ Northwestern Polytechnical University \\ Jin-Jie Ni \\ Northwestern Polytechnical University
}

DOI:

10.21203/rs.2.16792/v1

\section{SUBJECT AREAS}

Bioinformatics

\section{KEYWORDS}

Long noncoding RNA, alignment-free, multimodal learning, deep learning 
Abstract

Background: Long non-coding RNAs (IncRNAs) play crucial roles in diverse biological processes and human complex diseases. Distinguishing IncRNAs from protein-coding transcripts is a fundamental step for analyzing IncRNA functional mechanism. However, the experimental identification of IncRNAs is expensive and time-consuming.

Results: In this study, we present an alignment-free multimodal deep learning framework (namely IncRNA_Mdeep) to distinguish IncRNAs from protein-coding transcripts. LncRNA_Mdeep incorporates three different input modalities (i.e. OFH modality, k-mer modality, and sequence modality), then a multimodal deep learning framework is built for learning the high-level abstract representations and predicting the probability whether a transcript is IncRNA or not.

Conclusions: LncRNA_Mdeep achieves $98.73 \%$ prediction accuracy in 10 -fold cross-validation test on human. Compared with other eight state-of-the-art methods, IncRNA_Mdeep shows 93.12\% prediction accuracy independent test on human, which is $0.94 \% \sim 15.41 \%$ higher than that of other eight methods. In addition, the results on 11 cross-species datasets show that IncRNA_Mdeep is a powerful predictor for identifying IncRNAs. The source code can be downloaded from https://github.com/NWPU903PR/IncRNA_Mdeep.

\section{Full Text}

Due to technical limitations, full-text HTML conversion of this manuscript could not be completed. However, the manuscript can be downloaded and accessed as a PDF. Figures 


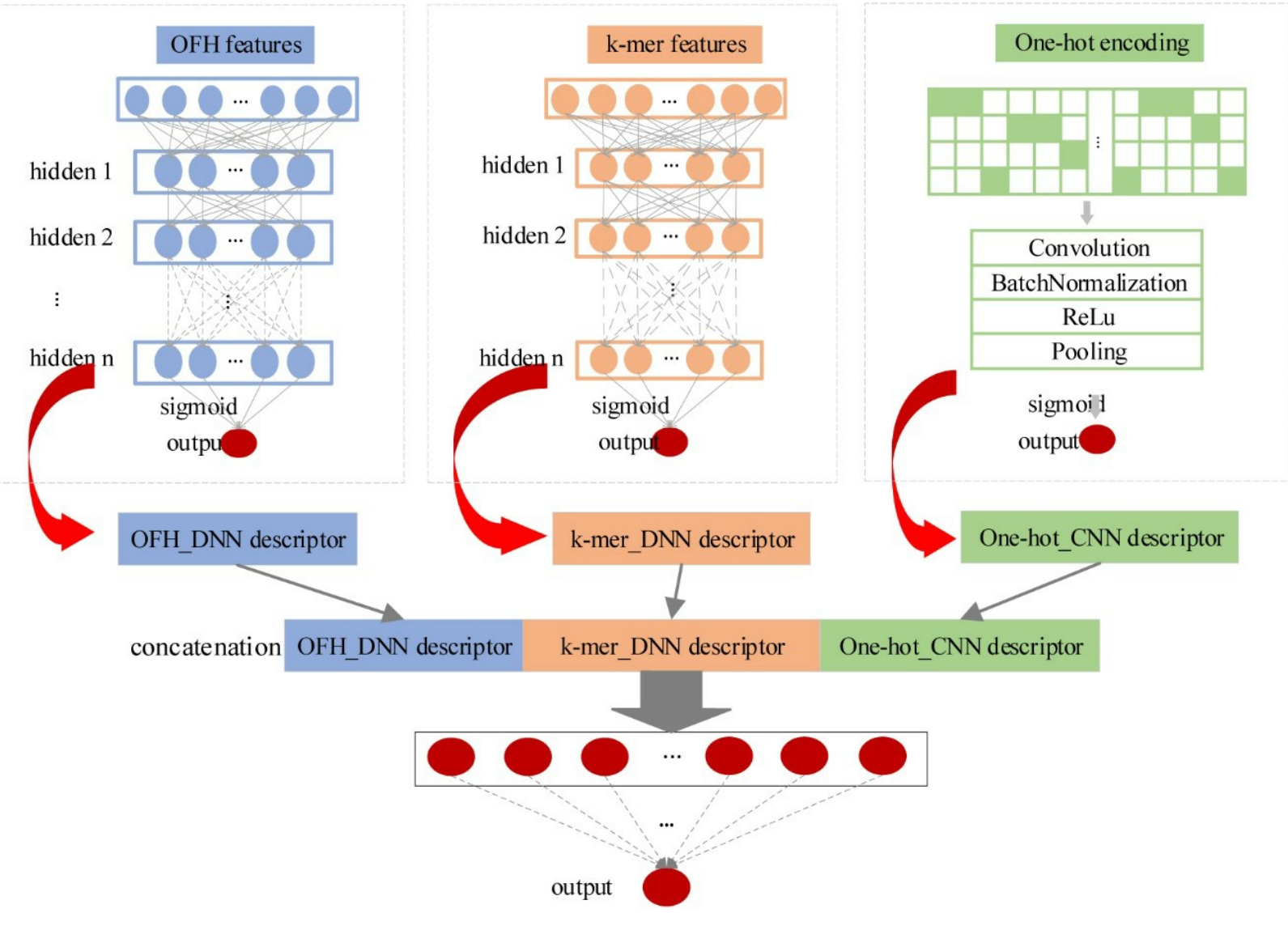

Figure 1

Overview of IncRNA_Mdeep
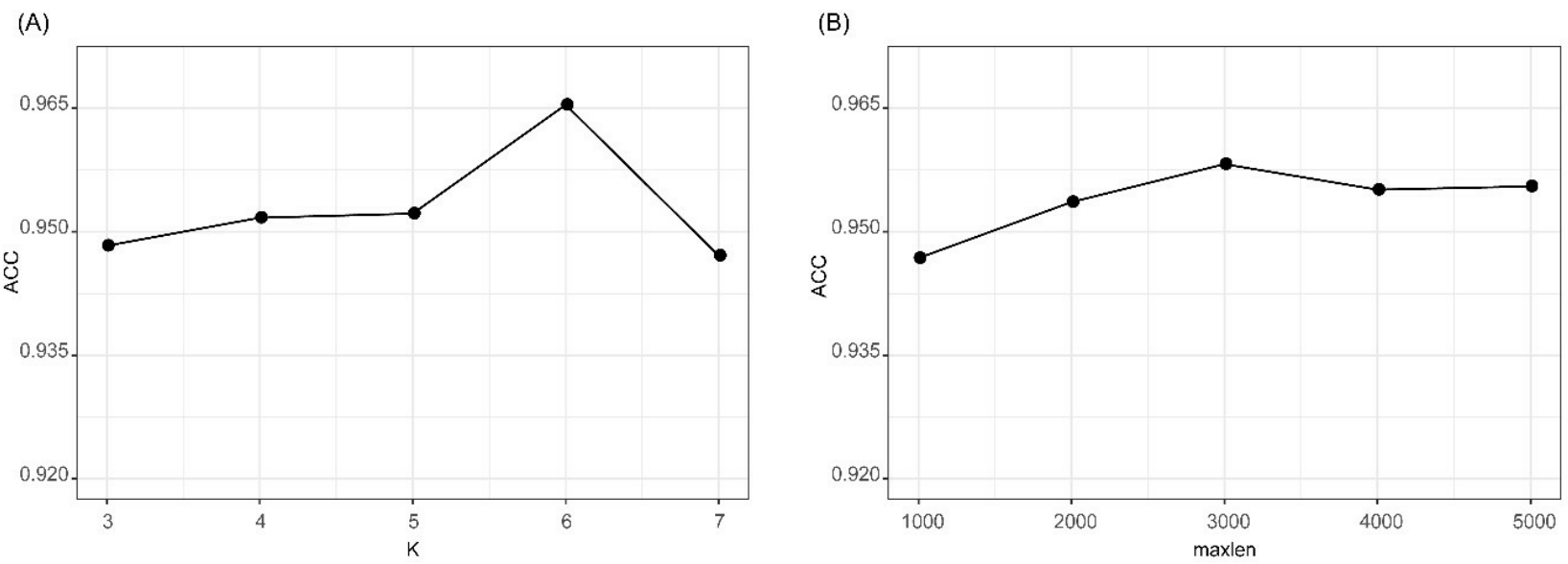

Figure 2

Results of k-mer_DNN and One-hot_CNN with different parameters. (A) Accuracy of kmer_DNN with different k value. (B) Accuracy of One-hot_CNN with different maxlen value. 
This is a list of supplementary files associated with this preprint. Click to download.

Additional file 1.tif

Additional file 2.docx 